\title{
Reseña de artículo: corresponsabilidad e interculturalidad en salud pública funciones esenciales de la salud pública
}

\author{
Co-responsibility and Intercultural in Public Health \\ ${\text { Cruz-Olvera }{ }^{1}}^{1}$
}

\begin{abstract}
:
The article emphasizes a new concept in the theoretical and application field of public health, this was generated seeking to appropriate both government and civil society in the pursuit of the common good understood as public health, the health of the Mexican population and that has application anywhere in the world. Aim. Promote the dissemination of the theory of co-responsibility and intercultural public health with educational strategies. Methodology. Systematic review and critical analysis that seeks to analyze the state of the art in terms of co-responsibility, education and multiculturalism. Results. The approach of vulnerable communities will serve to detect the real needs of these, as well as reflect the people who live and act according to their culture and conditions that justify an exercise of co-responsibility where the change of cultural influence in development is positively seen, as well as as an implementation of the theory of co-responsibility in public health. Conclusion. Public health co-responsibility represents today an opportunity for the boost that is required in health at the national level and a need for the incoming government in the country.
\end{abstract}

Keywords:

Values-attributes, governmental decisions, corruption, anti-values, essential functions of public health, quality of health care

Resumen:

El artículo hace hincapié en un nuevo concepto en el ámbito teórico y aplicativo de la salud pública, este fue generado buscando apropiar tanto a gobierno como a la sociedad civil en la búsqueda del bien común entendido como la salud pública, la salud de la población mexicana y que tiene aplicación en cualquier parte del mundo. Objetivo. Promover la difusión de la teoría de la corresponsabilidad y la salud publica intercultural con estrategias educativas. Metodología. Revisión sistemática y análisis crítico que busque analizar el estado del arte en materia de corresponsabilidad, educación y multiculturalidad. Resultados. El enfoque de comunidades vulnerables servirá para detectar las necesidades reales de estos, así como reflejar las personas que viven y actúan de acuerdo a su cultura y condiciones que justifiquen un ejercicio de corresponsabilidad donde se vean positivamente el cambio de influencia cultural en el desarrollo, así como una implementación de la teoría de la corresponsabilidad en salud pública. Conclusión. La corresponsabilidad en salud pública representa hoy en día una oportunidad para el impulso que se requiere en salud a nivel nacional y una necesidad para el gobierno entrante en el país.

Palabras Clave:

Corresponsabilidad, salud pública, interculturalidad, bien común

\section{Introducción}

La participación de la comunidad en la preservación o mejora de su estado de salud implica un constante ejercicio de corresponsabilidad; algunas veces el gobierno promueve servicios o propuestas para mitigar los daños en salud pública, pero la comunidad en ocasiones no participa tanto como seria lo esperado. Las participaciones de las personas en el cuidado de la salud junto con el cuidado de la participación de las autoridades hacen de la corresponsabilidad un espacio trascendente de participación mutua, y a la vez de accesibilidad en la que la comunidad se encuentre integrada en la preocupación de su propio estado de salud, por lo que la participación de ambas partes se hace evidente. Los programas y políticas contienen en esencia un doble propósito que es el de prestar atención y solucionar los problemas existentes en salud pública y que enfrenta actualmente las comunidades en el que la interacción de la infraestructura, recursos humanos y recursos financieros son tres elementos que deberán estar organizados para llevar a cabo las políticas implementadas para obtener los logros deseados y que

${ }^{1}$ Autor de Correspondencia. Estudiante de la Maestría en Salud Pública. Instituto Elise Freinet, Pachuca de Soto. México. Email: 
finalmente se traduce en el bienestar de la población como objetivo.

El papel de la población en el cuidado de su propia salud es indiscutible. El propósito inicial de la estrategia de atención primaria en la salud fue lo que se estableció en todo el mundo para el año 2000, alcanzando un nivel de salud que permitiera desarrollar una economía productiva y que dos de sus principios básicos eran lograr la accesibilidad y cobertura universal en salud con un compromiso, participación y auto sostenibilidad de individuos y de comunidades (Decálogo de Alma Ata, 1978)

Durante años, el gobierno representado por los servicios de salud ha sido responsable de ir proporcionando información a sus usuarios, olvidando que informar no es educar y esto es una prueba clara por el personal mismo de salud, que, si bien es el sector de la población la que este mejor informada sobre un tema será la población con menos problemas en salud, adoptando estilos de vida saludables con la única intención de que las estrategias establecidas sean exitosas.

Las estrategias básicamente educativas se basan en la comunicación, la cual está determinada por la cultura que adquirimos y es ahí, donde encontramos el termino de interculturalidad, definido como $\ll$ el proceso de comunicación e interacción entre personas y grupos en donde en donde no se permite tener a un grupo cultural por encima de otro, sino por el contrario, favoreciendo en todo momento la integración y convivencia entre culturas $>>$.

La interculturalidad debe ser entendida como una política, un proyecto social, epistémico y ético dirigido a la trasformación socio-histórica, basada en la construcción de una sociedad radicalmente diferente. La interculturalidad genera una cultura de solidaridad compartida, con apoyo mutuo y estrecha colaboración.

La corresponsabilidad e interculturalidad representan un gran desafío, que consiste en explorar la efectividad de los programas generados por el gobierno y el uso de los servicios de salud por los usuarios, mediante un modelo teórico para ser corresponsable al ciudadano con su gobierno. Un punto muy importante en esta situación es tener como idea principal la integración de la comunidad para generar un bien común y que el estado de salud resulte accesible con políticas y programas públicos que busquen mejorar el sistema de salud con acceso universal con equidad, calidad y justicia social.

Por lo que, para que se produzca un verdadero cambio, las prácticas de salud y los programas deberán ser reorientados a través de un compromiso mutuo entre el gobierno y la comunidad.

\section{Discusión}

La aplicación de programas de salud con estrategias en la prevención de muchas enfermedades, es para mejorar no solamente la calidad de vida, sino los estilos que pueda llevar a cabo una comunidad, ya que esto generaría un mayor impacto en su salud, primero en lo individual y luego en lo colectivo.

Sin embargo, una de las limitaciones corresponde a que los usuarios no cuentan con una educación, acceso y demanda alta de servicios de salud, mientras que la limitación que involucra al gobierno, es que los destinos de los recursos financieros deberían estar para cubrir a zonas de gran proporción con la presencia de determinantes y factores condicionantes que en un momento dado conduzcan a la mala salud.

Nuestra población muere en su mayoría por casusas prevenibles y que los programas asociados a la prevención en ocasiones están limitados por simples cambios en el comportamiento y que el ser humano en la actualidad se ve obligado a un mayor estudio para cambiar el concepto que tenemos con respecto a la salud individual y colectiva, no olvidando el bienestar social y los estilos de vida.

Es importante que el conocimiento debe desarrollarse con la curiosidad del investigador, en este caso de la población misma, y que gracias a esto nos ha llevado a generar nuevas actitudes con respecto a la educación para la salud pública.

La educación de las personas en los principios de higiene personal, así como la organización de los servicios médicos y de enfermería, hacen que el diagnóstico y tratamiento preventivo de las enfermedades tengan que asegurarse a todas las personas para un nivel de vida adecuado para la conservación de su salud, de tal manera, que el individuo esté en condiciones de disfrutar su derecho natural de salud y longevidad.

Es muy importante que, para lograr una reducción en la presentación de las enfermedades, existe una brecha entre los programas y la participación de las comunidades, la pregunta es ¿Por qué si los programas existen la población no participa? Esto se debe a que algunas de las estrategias que se utilizan no se entienden, o se les da poca importancia, porque no hacen entender su utilidad.

Dado que los programas creados por el gobierno deben basarse en las verdaderas necesidades de la población, se debe buscar que lleven a las personas a tomar posesión de su estado de salud, en donde la importancia del autocuidado para prevenir enfermedades siempre será el objetivo primordial y que por consecuencia la vida de ese individuo, será con una mejor calidad; pero sin olvidar que para ello deberá participar activa y conscientemente.

Cabe mencionar que varias estrategias y políticas nacionales, tiene presumido en sus objetivos el ser competentes y prometedoras, sin embargo, la realidad bajo la cual vive el mexicano es otra.

La transición epidemiológica ha llevado a la mutación de morbilidad y mortalidad de enfermedades infecciosas por la de enfermedades crónicas. Vivimos rodeados de muchas patologías en cuanto a alimentación, salud visual, auditiva, ecológica y educativa en donde los ambientes desafortunadamente han sido los actores adecuados para las enfermedades crónicas degenerativas.

\section{Análisis crítico}


Si bien es cierto, que en México no se ha logrado una verdadera transición epidemiológica es porque seguimos teniendo casos de morbilidad y peor aún, de mortalidad por enfermedades infecto contagiosas, ya que actualmente sigue habiendo defunciones en nuestro país por enfermedades diarreicas agudas e infecciones respiratorias altas en niños menores de cinco años, así como muertes maternas, casos de cólera por solo mencionar algunos, y que solo traducen que no hemos tenido un apego a las estrategias y políticas en salud que ha planteado el sistema nacional de salud a través de las reformas que ha sufrido durante los últimos años, y que para México dentro de los objetivos del milenio que le fueron trazados desde el año 2000 y que de los cuales en cuanto a salud se tenía que reducir tasas de mortalidad infantil, la razón de muerte materna, y casos de desnutrición; elevando el nivel de educación de las comunidades y que México tuvo 15 años para trabajar en ello, finalmente se llegó al 2015 y no se cumplieron los objetivos del milenio, por lo que México sigue siendo un país subdesarrollado y que hablando objetivamente nunca lograra una transición epidemiológica verdadera a diferencia de países de primer mundo, si las malas prácticas de higiene continuando existiendo de manera individual y aunado a ello la participación de las comunidades no sea tan eficiente para dicha situación, por lo que se tiene mucho camino aun por recorrer y que la educación es la base fundamental para las nuevas generaciones y que se logre un verdadero cambio.

Si bien, las estrategias en salud que actualmente tiene el sistema nacional de salud son buenas creo que cuando verdaderamente se unifiquen los servicios de salud en un solo organismo y que se termine con muchas practicas burocráticas y las establecidas por los subsistemas de salud, solo así se podrá garantizar el regresarle el nivel de salud a los individuos y algo fundamental es no olvidar que la medicina preventiva es la piedra angular en el nivel de salud de las comunidades en donde la promoción deberá ser el brazo fuerte para las buenas prácticas de higiene en las comunidades. Sin olvidar los fundamentos que se establecieron en la comunidad de Alma Ata a través del decálogo, el cual surgió ante una necesidad fuerte y global que se tenía sobre por epidemias en varias regiones del mundo y que actualmente nos hemos olvidado de esa atención primaria a la salud y solo realizamos medicina curativa o de rehabilitación.

\section{Opinión}

En lo personal, la salud es responsabilidad no solo del personal que labora en salud, sino el autocuidado y las buenas prácticas que se lleven a cabo de manera individual hará que esa comunidad sea una población con un nivel de salud optimo y recordemos que una comunidad sana, es una comunidad productiva, lo cual finalmente impacta en el desarrollo socio - económico y cultural que tiene un país, ya que actualmente estamos ante una globalización y que cualquier epidemia que afecte a una comunidad, tarde que temprano afectara al resto del país. En lo particular, si un padre de familia se encuentra enfermo y es el único sustento económico para su familia, desde ese momento empieza un caos social, por lo que el recuperar lo antes posible el nivel de salud de ese individuo será pieza fundamental para el desarrollo de los integrantes de su familiar, y si esto lo traspolamos a un brote por las malas prácticas de higiene que pueda desencadenar una sola persona, afectará inmediatamente al resto de la comunidad.

\section{Conclusiones}

Para una corresponsabilidad sanitaria equitativa junto con las políticas generadas por el gobierno con estrategias educativas exitosas, deberán fusionarse con el concepto de interculturalidad, que permita a los profesionales de la salud encontrar coincidencias, facilitando la comunicación entre la población con prácticas que motiven hacia la acción y adopción de estilos de vida saludables, lo que mejorara no solo la calidad de vida de manera individual, sino al mismo tiempo en toda una comunidad.

La corresponsabilidad es un compromiso tanto de la comunidad como del gobierno con el fin de restablecer el bienestar con el autocuidado y calidad de la atención de una población.

La corresponsabilidad en salud pública representa hoy en día una oportunidad para el impulso que se requiere en salud a nivel nacional y una necesidad para el gobierno entrante en el país.

\section{Referencia}

1. Cortés Ascencio Sandra Yazmín, Jesús Carlos Ruvalcaba Ledezma, Avila Domínguez Rosangela, Torres Guevara Rosa Silvana, Calderón Rodríguez Nelly Patricia, Pérez Torres Lucia Vanessa, Barragán López Norma, Cobián Díaz Mariela Lizbeth, and Reynoso Vázquez Josefina, "Co-responsibility and Intercultural in Public Health." American Journal of Public Health Research, vol. 5, no. 6 (2017): 174-180 DOI. 10.12691/ajpjr-5-6-2 